\title{
Effects of Pacing When Using Material Handling Manipulators
}

\author{
Maury A. Nussbaum, Virginia Polytechnic Institute and State University, Blacksburg, \\ Virginia, and Don B. Chaffin, University of Michigan, Ann Arbor, Michigan
}

\begin{abstract}
Common manipulator-assisted materials handling tasks were performed in a laboratory simulation at self-selected and faster (paced) speeds. The effects of pacing on peak hand forces, torso kinematics, spine moments and forces, and muscle antagonism were determined, along with any influences of several task variables on these effects. The faster trials were performed $20 \%$ more rapidly than the self-paced trials. It was found that (a) achieving this level of performance required $\sim 10 \%$ higher hand forces and 5\%-10\% higher torso moments, (b) consistent torso postures and motions were used for both speed conditions, and (c) the faster trials resulted in $\sim 10 \%$ higher spine forces and $\sim 15 \%$ higher levels of lumbar muscle antagonism. On whole, these results suggest a higher risk of musculoskeletal injury associated with performance of object transfers at faster than self-selected speeds with and without a manipulator. Further analysis provided evidence that the use of manipulators involves higher levels of motor coordination than do manual tasks. Several implications regarding the use of material handling manipulators in paced operations are discussed. Results from this investigation can be used in the design, evaluation, and selection of material handling manipulators.
\end{abstract}

\section{INTRODUCTION}

Numerous sources have demonstrated an association between manual materials handling and work-related lost time attributable to musculoskeletal injury (e.g., Ayoub, 1982; Chaffin \& Park, 1973; Marras et al., 1993). In attempts to control these types of disorders, mechanized assistive devices have been increasingly incorporated into material transfer processes. Material handling systems may be broadly classified (Sowden, Jimmerson, Joseph, \& Chaffin, 1998) as positioners (lift tables, conveyors), which are used to place or orient an object, and manipulators (arms, hoists), which are used to move and/or support an object and which are the focus of the present work. With the use of manipulator assistance, the decrease in gravitational loads that the worker must counteract during manual material handling should lead to a decrease in the ensuing musculoskeletal stresses (e.g., muscle and spine forces). However, there are anecdotal reports that when mechanical devices are available they are not used, often because of concerns related to productivity. It has also been suggested (Woldstad \& Chaffin, 1994; Woldstad \& Reasor, 1996) that the dynamics and substantial inertia of most manipulators have been ignored in the workplace specification of these devices.

Material transfer tasks can often require additional time for completion when a manipulator is used. Because these increases in performance times lead to decreases in productivity, manipulators are often rejected even after installation in manufacturing facilities. In the absence of quantitative evaluations of the effects of manipulators and pacing, the facility planner does not have sufficient evidence to weigh the opposing effects: fatigue, injuries, or both 
from not incorporating manipulators versus slowdowns from using manipulators.

Previous investigations and biomechanical modeling of manipulator-assisted materials handling tasks have focused primarily on the static gravitational component. As most of the static component (i.e., weight) is reduced when using a manipulator, the remaining external loads come primarily from body segment dynamics and inertial dynamics of the manipulator + object mass. Also, manipulator-assisted operations often result in asymmetric body motions, suggesting that complex patterns of torso muscle activity, including antagonism, are probably present. Thus, improved realism in the evaluation and modeling of manipulatorassisted tasks requires the use of dynamic three-dimensional biomechanical modeling, including an accurate methodology for ascertaining dynamic muscle and spine forces.

A limited number of studies have investigated physical stresses associated with the use of manipulators. In one study using an overhead cable-hoist system, Woldstad and Chaffin (1994) found that inexperienced participants performed rapid motions with high peak levels of push $(200-500 \mathrm{~N})$ and pull $(150-300 \mathrm{~N})$ forces. Further, these high hand forces were only minimally influenced by experimental manipulation of load, distance, target width, and friction. Resnick and Chaffin (1996) investigated symmetric and twisting tasks using an articulated arm to move objects of 0-68 kg. Results showed that higher object masses and task asymmetry increased peak hand forces. Psychophysical ratings were only moderately related to hand forces, suggesting that measured hand forces do not adequately represent limiting physical stresses during manipulator use and, thus, that the risk of musculoskeletal injury may not be controlled by voluntarily limited exertions.

Given the paucity of detailed biomechanical evaluations of material handling manipulators and, in particular, the unknown influence of task execution speed on spinal stresses, we performed an experimental study to evaluate the effects of pacing when using two types of material handling manipulators. The first experimental hypothesis was that more rapid object transfers when using a material han- dling manipulator would be associated with larger hand forces, altered trunk kinematics, increased spine forces, and increased muscular antagonism. The second hypothesis was that pacing-induced increases in these dependent measures would be influenced by specific task parameters: (a) type of manipulator used, (b) height at which the object is transferred, (c) asymmetry of the task motions, and (d) mass of the transferred object.

\section{METHODS}

This initial investigation focused on what were considered elemental activities involved in the acquisition, transfer, and placement of material. Experimental tasks simulated realistic industrial activities to allow investigation of transitional biomechanical loads occurring during initiation and termination of short-distance transfer movements. This focus was justified because of the strong likelihood of peak musculoskeletal loads arising from accelerations of body segments, the objects being transferred, and the manipulators themselves. The set of experimental tasks and object masses encompassed a range of commonly adopted postures and load positions used in industrial material handling tasks.

\section{Conditions Studied}

Experimental tasks were performed to evaluate the effects of four independent task variables:

1. Method. Objects were moved manually (manual), with a pneumatically balanced articulated arm (arm) or with a pneumatic-powered hoist (hoist) on a moveable overhead bridge rail (Figure 1).

2. Task. Objects were acquired from and placed to midshank or elbow height. The elbow height transfers were performed in both the midsagittal (Figure 1) and frontal planes.

3. Mass. Object masses of 10, 20, and $40 \mathrm{~kg}$ were used (40 kg with manipulator assistance only).

4. Speed. Two transfer paces were studied. A preferred, or unpaced, speed was simply that which the participants adopted, after practice, as comfortable. The participants were encouraged to find a personal balance between competing goals: "Work quickly in order to minimize transfer time, yet work slowly enough to minimize any physical discomfort in performing the tasks." The fast, or paced, speed 


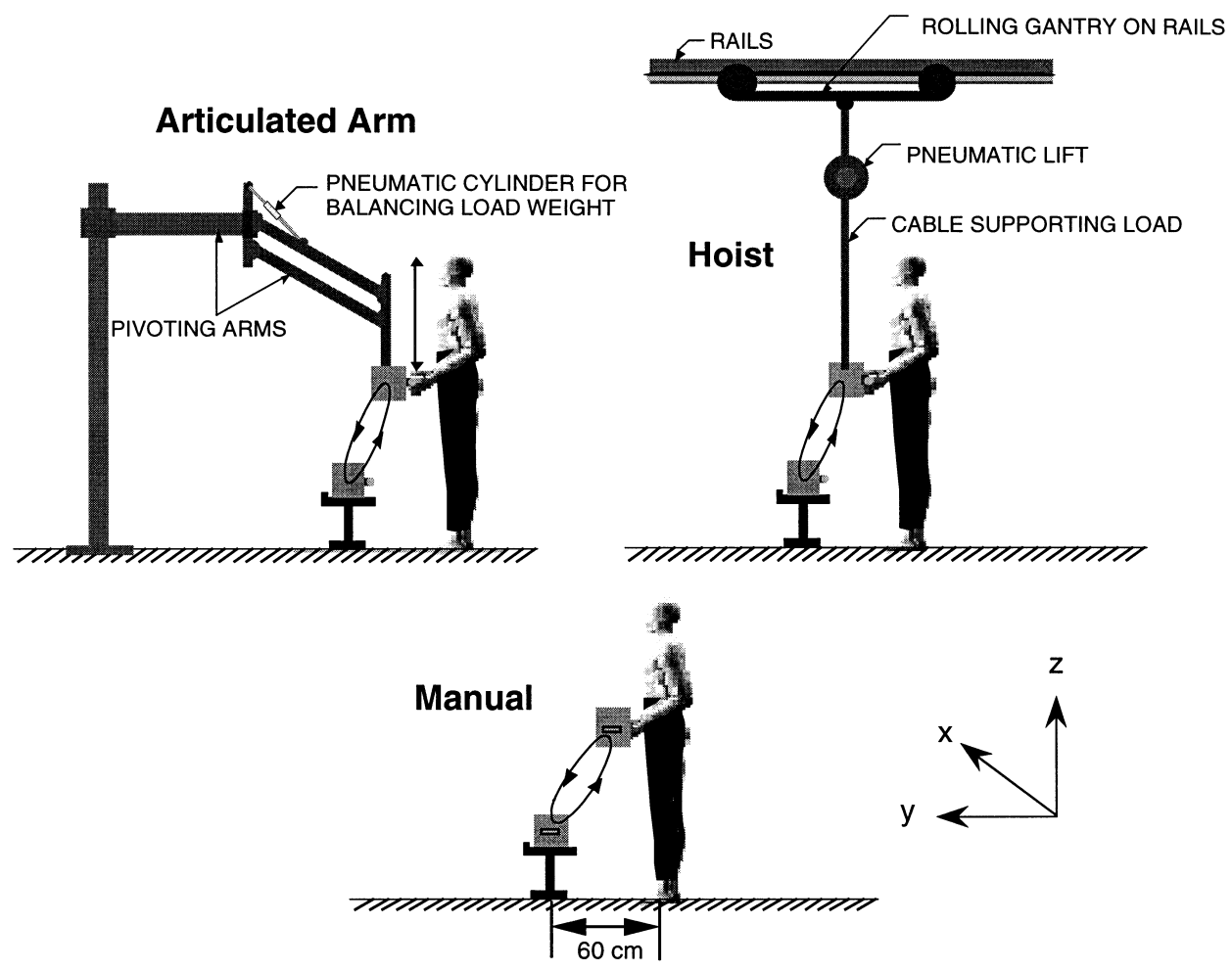

Figure 1. Illustration of object transfers. The figure (not to scale) shows the three methods used to transfer the objects in the sagittal plane to and from approximately midshank height. Participants moved the objects using handles attached to the front of the box when using arm and hoist and at the sides of the box during manual transfers.

was achieved by decreasing the allowable transfer time by $20 \%$ using repeated tones, and the participants performed several practice trials to accommodate to this faster pace.

\section{Testing Procedures}

The participants began each trial in a relaxed standing position with their arms hanging. The object (a wooden box) sat in front of the participant on a small platform, the height of which was set so that the center of the box was at either midshank $(36 \mathrm{~cm})$ or elbow height $(101$ $\mathrm{cm})$. With the exception of handle position (Figure 1), the manual and manipulator-assisted trials were identical. Sagittal plane transfer trials consisted of a half-step forward to grasp handles attached to the object (acquire) and a halfstep backward that ended with the object held in a position (typically near elbow height) to allow potential movement over a moderate distance. After a brief pause, participants again took a half-step forward and set the object back on the platform (place). Frontal plane (or lateral) transfer trials started with the participant midway between two platforms and $\sim 0.6 \mathrm{~m}$ behind them. These transfers required twisting and/or laterally bending to grab the box on the right-hand platform, movement to the lefthand platform, a brief pause, and then a reverse movement returning the box to the right-hand platform.

All trials required 4 to $8 \mathrm{~s}$ for completion, and at least $1 \mathrm{~min}$ of rest was given between trials. The order of trials was partially randomized in that a faster trial always followed the corresponding unpaced trial and object placement always followed acquisition. The trials were randomized with respect to the method, task, and mass.

\section{Participants}

The 10 participants ( 5 men and 5 women) were university students and completed an 
informed consent procedure approved by the University of Michigan Human Subjects Review Committee. The participants were relatively young (range $=20-30$ years) and had a wide distribution in stature $($ mean $=173 \mathrm{~cm}$, range $=$ $154.3-194.4 \mathrm{~cm}$ ) and body mass (mean = 69 $\mathrm{kg}$, range $=54.3-97.9 \mathrm{~kg}$ ).

\section{Measurements and Biomechanical Models}

Posture was monitored using surface markers adhered to the participant over bony landmarks at the wrist, elbow, shoulder, thoracic spine, and pelvis. Markers coordinates were obtained at $25 \mathrm{~Hz}$ (pilot work indicated minimal signal power $>10 \mathrm{~Hz}$ ), and joint center locations were determined using a heuristic transformation from the surface marker locations (Nussbaum, Zhang, \& Chaffin, 1999). Markers attached to the spine and pelvis were used to estimate torso angles, defined relative to a pelvic local coordinate system. Differentiation of torso angles, using finite differences (Winter, 1990), yielded torso angular velocities. A single triaxial transducer (AMTI SRMC3A-6-1000) attached between a horizontal handle and the box (Figure 1) measured hand forces (total of both hands) during manipulator-assisted trials. Hand forces during manual trails were estimated from the box kinematics.

Myoelectric activity of the torso musculature was monitored using surface electrodes; these were attached as described by Lavender, Tsuang, Andersson, Hafezi, and Shin (1992) and McGill (1992) over the thoracic and lumbar components of erector spinae, internal and external obliques, rectus abdominis, and latissimus dorsi. Raw electromyographic (EMG) signals were hardware preamplified, rectified, and filtered. The processed EMGs and hand force data were sampled at $50 \mathrm{~Hz}$. All data were low-pass $(\sim 10 \mathrm{~Hz})$ software filtered, following which the EMG and hand force data were resampled and synchronized with joint coordinate data.

Lumbar muscle forces were estimated using a dynamic EMG-based model (Nussbaum \& Chaffin, 1998) that is a modification of earlier work (Granata \& Marras, 1993, 1995a). The model is calibrated for each participant using pre-experimental calibration trials and yields estimates of muscle force based on normalized
EMG records. Spine forces were computed from predicted muscle forces using participantand posture-specific muscle moment arms and lines of action (Nussbaum \& Chaffin, 1996).

\section{Comparison Metrics}

Measures quantified in this study involved task duration, hand forces, torso kinematics, reactive spinal forces, and a derived metric of muscular antagonism. Individual experimental trials were separated into their respective acquire and place phases, as described earlier. Task duration (movement time) was determined from the marker location files by tabulating times between the start and end of object motion. The influence of pacing on performance $(\Delta t)$ was quantified from the task execution times during paced $\left(t_{\text {fast }}\right)$ and unpaced $\left(t_{\text {preferred }}\right)$ trials:

$$
\Delta t=\frac{\left(t_{\text {preferred }-} t_{\text {fast }}\right)}{t_{\text {preferred }}} \times 100 \%
$$

Hand force data were processed to obtain the peak values of the resultant (external) hand force vector magnitude, with reported values comprising the total force exerted on the object by both hands. Peak values of torso angles and angular velocities were determined about three axes defined by the pelvic local coordinates. Given observations that all torso sagittal angles were in flexion, and with an assumed equivalence between right and left lateral bending and twisting, all torso kinematic data were transformed to their absolute values prior to determining peaks. Peak external spine moments and reactive spine forces (compression and anteroposterior and lateral shear) were determined for the L3-L4 motion segment and relative to a posture-specific local coordinate system at this level. Similar to torso kinematics, all lumbar moments and forces were transformed to absolute values before determining the peak. Peak values of all biomechanical measures (including muscular cocontraction, described later) were obtained separately for the two phases (i.e., during the acquiring and placing of the box off and on the shelf).

A metric was derived to quantify the extent of torso muscle antagonism and is based on comparison of EMG-based and optimizationbased estimates of spine compression from the 
algorithm of Bean, Chaffin, and Schultz (1988). An optimization-based simulation of the experimental trials showed that the external spine moments could be equilibrated using only the posterior musculature (erectores spinae and latissimus dorsi). Because this optimizationbased approach implicitly minimizes spine compression, the set of muscle forces so generated can be considered a "minimal" set with respect to spine compression. Thus any activity in the anterior musculature is here considered excess or antagonistic relative to the optimizationbased estimates. The difference between optimization-based and EMG-based compression estimates, $\Delta F(\mathrm{~N})$, quantifies this excess. An antagonism metric $(A M$, in $\mathrm{N})$ was derived for each phase of each trial:

$$
A M_{\text {peak }}=\max \left[\Delta F_{i}\right]_{i=1}^{n}
$$

Thus, the peak value of the antagonism metric $\left(A M_{\text {peak }}\right)$ indicates the maximum excess in compression predicted from the EMG-based model across the $n$ samples in a given trial and phase of motion.

\section{Statistical Analysis Methods}

The set of dependent measures (see Figure 1 for axis conventions) included peak values of hand forces $(H F \mathrm{~s})$; torso angles $(A x, A y, A z)$, angular velocities $(V x, V y, V z)$, and external L3-L4 moments $(M x, M y, M z)$ in the sagittal, frontal, and horizontal planes; spine lateral shear $(F x)$, anteroposterior shear $(F y)$, and compression $(\mathrm{Fz})$ forces; and muscular antagonism $(A M)$. The overall significance of pacing on these dependent measures was ascertained using a paired $t$ test for the hypothesis that the effect was $>0$. In addition to the four transfer conditions (method, task, mass, speed), the analysis included transfer phase as a potential effect. A preliminary multivariate analysis of variance (MANOVA) was performed using all main effects and two-way interactions and Wilks's lambda test for significance. Subsequent analyses of variance (ANOVAs) were used to examine effects of the task variables on specific dependent measures and using subsets of the data to compare (a) sagittal plane transfers at midshank and elbow height, (b) sagittal and frontal plane transfers at elbow height, and (c) frontal plane transfers at elbow height using different object masses. Each ANOVA was based on an underlying model that included all main and two-factor interactions, with subject as a random blocking variable. Statistical significance was determined using a criterion of $p<.1$ because of the preliminary nature of the study, the relatively small sample size, and large intra- and intersubject variances observed in previous work (Nussbaum, Chaffin, \& Baker, in press; Nussbaum, Chaffin, Stump, Baker, \& Foulke, 1998).

Participants were not always successful in achieving reduced task execution times at the desired 20\% level. Paced trials with $\Delta t<5 \%$ $(\sim 15 \%$ of trials) were not included in the analysis. For the remaining 160 trials, $\Delta t$ averaged $20.17 \%(S D=9.59)$. Of note is the fact that the dependent measures were not consistently related to $\Delta t$, nor was $\Delta t$ significantly different for any of the experimental conditions. Thus normalization of dependent measures using the magnitude of the pacing effect, yielding an indication of the change in the dependent measures per unit $\Delta t$, was not performed.

\section{RESULTS}

\section{Overall Effects of Pacing}

Initial MANOVAs revealed several significant effects of the task conditions on the dependent measures as a whole. All main effects were highly significant with $p<.0001$. Significant two-way interactions included Method $\times$ Height $(p<.0001)$, Method $\times$ Mass $(p<.0001)$, Speed $\times$ Method $(p<.05)$, and Phase $\times$ Height $(p<$ $.0001)$. The Speed $\times$ Task interaction approached significance, with $p=.17$.

Results compiled across all experimental conditions indicated the overall magnitude of pacing effects on the dependent measures (Tables 1 and 2, Figure 2). Peak hand forces were roughly $10 \%$ larger during the paced trials. Although pacing was associated with significant increases in peak sagittal torso angles $(A x)$, the actual change was small. The effects of pacing were significant for all three peak angular velocities, with values increasing 10\%-15\% 
TABLE 1: Peak Hand Forces (HFs) and Peak Torso Kinematics in Unpaced and Paced Trials

\begin{tabular}{|c|c|c|c|c|c|c|c|}
\hline \multirow[b]{2}{*}{ Measure } & \multirow[b]{2}{*}{$H F(N)$} & \multicolumn{3}{|c|}{ Torso Angle $\left({ }^{\circ}\right)$} & \multicolumn{3}{|c|}{ Torso Angular Velocity ( $\% / s)$} \\
\hline & & $A x$ & Ay & $A z$ & $V x$ & Vy & Vz \\
\hline Unpaced & $\begin{array}{c}116.6 \\
(59.1)\end{array}$ & $\begin{array}{c}17.5 \\
(15.8)\end{array}$ & $\begin{array}{c}6.7 \\
(3.5)\end{array}$ & $\begin{array}{c}7.4 \\
(2.7)\end{array}$ & $\begin{array}{c}22.9 \\
(17.0)\end{array}$ & $\begin{array}{c}13.9 \\
(5.6)\end{array}$ & $\begin{array}{c}18.5 \\
(7.9)\end{array}$ \\
\hline Paced & $\begin{array}{c}127.8 \\
(57.4)\end{array}$ & $\begin{array}{c}18.0 \\
(16.7)\end{array}$ & $\begin{array}{c}7.0 \\
(3.9)\end{array}$ & $\begin{array}{c}7.4 \\
(3.0)\end{array}$ & $\begin{array}{c}25.5 \\
(19.1)\end{array}$ & $\begin{array}{c}16.0 \\
(6.7)\end{array}$ & $\begin{array}{c}21.1 \\
(8.4)\end{array}$ \\
\hline$p$ value & $<.0001$ & .08 & .12 & .35 & $<.0001$ & $<.0001$ & $<.0001$ \\
\hline
\end{tabular}

The first two rows present the mean $(S D)$ values of the dependent measures taken across all unpaced and paced trials, respectively. The significance of the overall pacing effect is given in the third row ( $p$ value from one-sided $t$ test that paired [paced-unpaced] values $>0$ ). See Methods for definitions and Figure 1 for axis conventions.

over baseline levels. Peak moments were larger during the paced trials in all three planes, although the average effect was 1-2 Nm. Peak spine forces were also larger during the paced trials, with both shear and compression forces increasing approximately $10 \%$ over baseline levels. The metric of antagonism $(A M)$ was high on average during the baseline trials and increased nearly $17 \%$ during the paced trials. Given these observations that pacing affected the magnitudes of the dependent measures, in the following analyses we examined the dependent measures in more detail and determined any influences (interactions) of other task variables. Results presented in the following sections are limited to the effects of pacing and its interaction with the remaining factors (see Nussbaum, Chaffin, \& Baker, in press, and Nuss- baum, Chaffin, Stump, et al., 1998, for details regarding the effects of method, task, and mass).

\section{Influence of Transfer Height}

Transfers from midshank and elbow levels (20-kg object) in the sagittal plane using the three methods were compared. Speed was significant as a main effect $(p<.01)$ on peak $H F$ s, as were the Speed $\times$ Method $(p<.1)$ and Speed $\times$ Height $(p<.1)$ interactions. Peak HFs were higher during paced $(153 \mathrm{~N})$ than unpaced $(138 \mathrm{~N})$ trials. Pacing caused the largest overall increases in $H F$ s when using either the arm or hoist (25 and $21 \mathrm{~N}$, respectively) in comparison with the manual method $(3 \mathrm{~N})$, and for transfers from midshank $(24 \mathrm{~N})$ versus elbow $(7 \mathrm{~N})$ height.

On average, peak $A x$ increased with pacing (from $30^{\circ}$ to $31^{\circ}$ ), though the difference was

TABLE 2: Peak Lumbar Moments, Spine Forces, and Muscular Antagonism in Unpaced and Paced Trials

\begin{tabular}{lcccccccc}
\hline & \multicolumn{3}{c}{ Lumbar Moment $(\mathrm{Nm})$} & & \multicolumn{3}{c}{ Lumbar Spine Force (N) } & \\
\cline { 2 - 3 } \cline { 6 - 8 } Measure & $M x$ & $M y$ & $M z$ & & $F x$ & $F y$ & $F z$ & $A M(N)$ \\
\hline Unpaced & 62.6 & 24.7 & 10.3 & & 115.4 & 160.2 & 1754 & 795 \\
& $(54.1)$ & $(16.2)$ & $(5.6)$ & & $(81.9)$ & $(129.5)$ & $(890)$ & $(486)$ \\
Paced & 65.6 & 26.6 & 11.7 & & 123.8 & 175.3 & 1923 & 927 \\
& $(56.9)$ & $(17.6)$ & $(6.0)$ & & $(67.8)$ & $(152.7)$ & $(903)$ & $(551)$ \\
p value & .0003 & $<.0001$ & $<.0001$ & & .079 & .001 & $<.0001$ & $<.0001$ \\
\hline
\end{tabular}

The first two rows present the mean $(S D)$ values of the dependent measures taken across all unpaced and paced trials, respectively. The significance of the overall pacing effect is given in the third row ( $p$ value from one-sided $t$ test that paired [paced-unpaced] values $>0$ ). See Methods for definitions and Figure 1 for axis conventions. $A M=$ antagonism metric. 

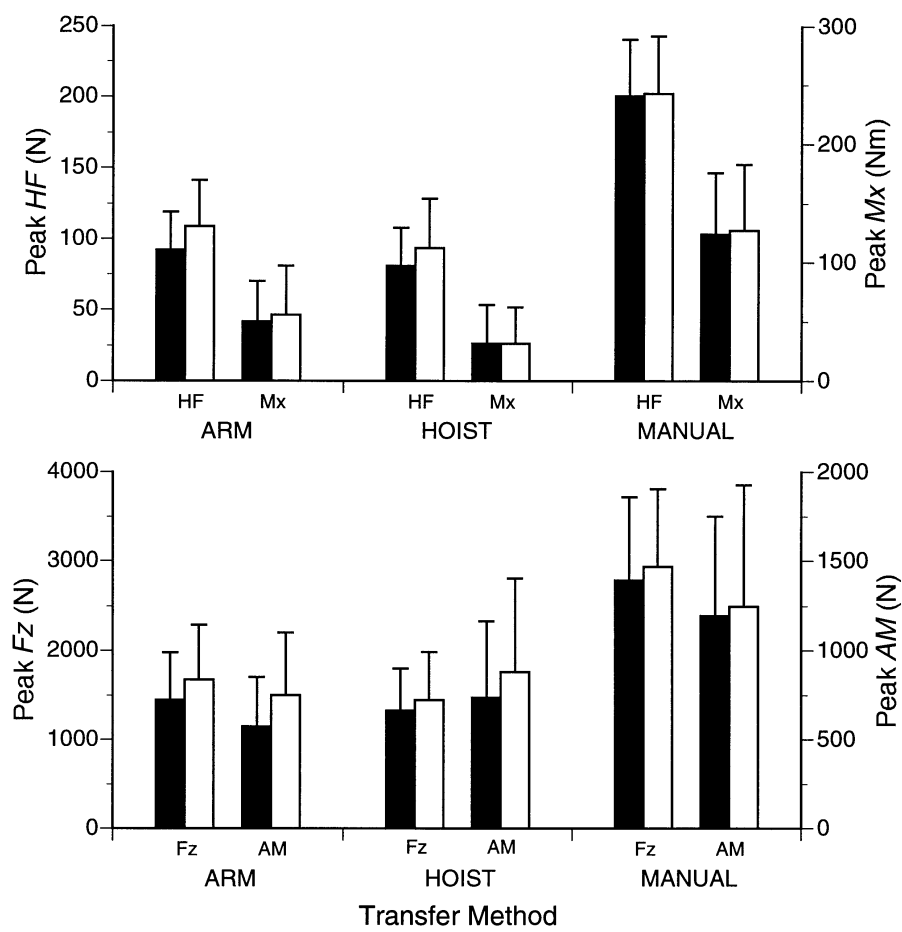

Figure 2. Illustration of the effects of pacing and transfer method on peak hand forces $(H F)$, sagittal plane moments $(M x)$, spine compression $(F z)$, and muscular antagonism $(A M)$. Results are compiled across all experimental conditions. Solid and empty bars indicate unpaced and paced trials, respectively.

not significant $(p=.28)$. Speed and its interactions did not have any other significant effects on the remaining kinematic measures, and no substantial or consistent trends were found. Pacing significantly $(p<.05)$ increased peak $M x$, whereas the Speed $\times$ Method interaction $(p=.14)$ approached significance. There were also significant speed effects on peak $F z(p<.1)$ and peak $A M(p<.05)$. None of these effects was significantly influenced by transfer height, although the increase in $A M$ with pacing tended $(p=.24)$ toward higher values for lifts at midshank $(297 \mathrm{~N})$ versus elbow (69 N) heights.

\section{Influence of Transfer Asymmetry}

Transfers at elbow level (20-kg object) in the frontal and sagittal planes using the three methods were compared. Speed was significant as a main effect $(p<.001)$ on peak $H F \mathrm{~s}$, as was the Speed $\times$ Method $(p<.05)$ interaction. Peak $H F$ s were higher during paced $(144 \mathrm{~N})$ than unpaced $(137 \mathrm{~N})$ trials, and pacing caused the largest overall increases in $H F$ s when using either the arm $(18 \mathrm{~N})$ or hoist $(7 \mathrm{~N})$ in comparison with the manual method $(1 \mathrm{~N})$. The effect of pacing was not influenced by the plane of motion $(p=.86)$.

Speed, as a main effect, was not significant for any of the kinematic measures $(p=$ $.46-.85)$. There were significant Speed $\times$ Plane interaction effects on both peak torso sagittal plane angles $(A x)$ and peak frontal plane velocities $(V y)$, with $p<.05$ and $p<.1$, respectively. Peak $A x$ values increased with pacing when transfers were performed in the sagittal plane but decreased for the asymmetric transfers. These pacing effects, however, were relatively small $\left(<1^{\circ}\right)$. On average, peak values of $V y$ decreased $6^{\circ}$ during the paced trials in the sagittal plane but increased $5^{\circ}$ in the asymmetric trials.

Pacing caused significant $(p<.001)$ increases in peak $M z$, on the order of $1.5 \mathrm{Nm}$. The interaction effects of Speed $\times$ Plane approached significance $(p=.14)$, wherein the increase in peak $M z$ with pacing was higher for the asymmetric $(2 \mathrm{Nm})$ than symmetric 
(1 Nm) transfers. Pacing was significant as a main effect for peak $F z$, increasing the values on average by $163 \mathrm{~N}$. The effect of pacing on muscular antagonism $(A M)$ approached significance $(p=.12)$, increasing from $797 \mathrm{~N}$ to 868 N. The effects of speed on spine forces and muscular antagonism were not influenced by the plane of transfer.

\section{Influence of Object Mass}

Frontal plane transfers at elbow level were compared with three different object masses and using the three methods. Speed was significant $(p<.05)$ as a main effect on peak $H F$, with unpaced and paced trials averaging $105 \mathrm{~N}$ and $114 \mathrm{~N}$, respectively. A Speed $\times$ Mass interaction was found to be significant $(p<.1)$ for $A x$, wherein the values of $A x$ showed larger increases with pacing for lighter masses. These angles, however, differed on the order of only $1^{\circ}$. Pacing had no other influences on hand forces or trunk kinematics. Speed had significant main effects on both $M y(p<.05)$ and $M z$ $(p<.01)$. Both moments were larger during the paced $(M y=32 \mathrm{Nm} ; M z=13 \mathrm{Nm})$ than unpaced trials $(M y=29 \mathrm{Nm} ; M z=12 \mathrm{Nm})$. A significant Speed $\times$ Method interaction effect $(p<.01)$ on $M z$ was found, although the magnitude of this effect was small $(\sim 1 \mathrm{Nm})$.

Speed did not have significant main effects on either $F x$ or $F y$. Peak values of $F x$, however, were significantly affected by the Speed $\times$ Method $(p<.1)$ and Speed $\times$ Mass $(p<.1)$ interactions (Figure 3). Pacing caused increases in $F x$ for arm and manual transfers $(24 \mathrm{~N}$ and $21 \mathrm{~N}$, respectively), whereas decreases were found for hoist transfers $(-10 \mathrm{~N})$. The effect of pacing on $F x$ was highly dependent on the load during manual transfers (Figure 3); pacing resulted in larger increases in $F x$ when the 10-kg mass was transferred $(27.9 \mathrm{~N})$, whereas a small decrease in peak Fx occurred with the $20-\mathrm{kg}$ mass. Although speed did not have a main effect on $F y$, there was a significant Speed $\times$ Mass interaction $(p<$ .1). Pacing resulted in a $\sim 20 \mathrm{~N}$ increase in peak $F y$ for transfers of 20 and $40 \mathrm{~kg}$ and a decrease
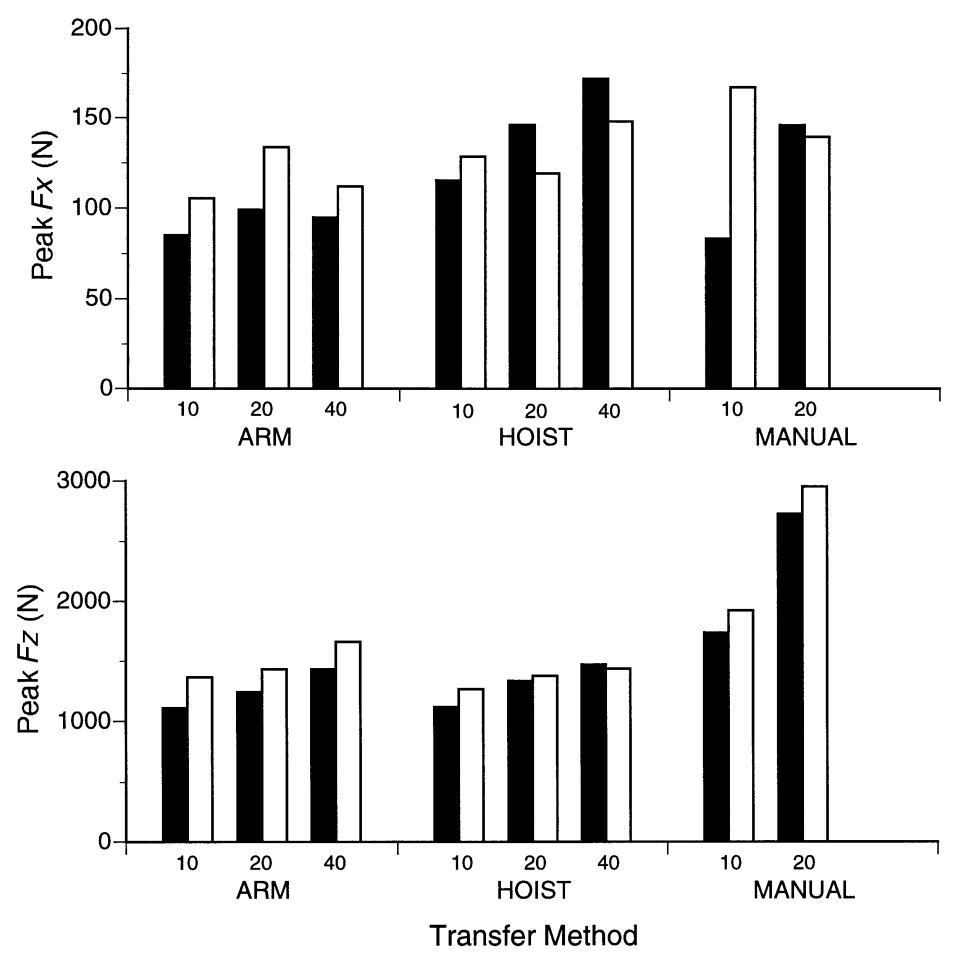

Figure 3. Illustration of the effects of pacing, transfer method, and object mass on peak spine lateral shear $(F x)$ and compression $(F z)$ forces. Solid and empty bars indicate unpaced and paced trials, respectively. Within each transfer method, the three different object masses (in kilograms) are listed. 
of $\sim 20 \mathrm{~N}$ for transfers of $10 \mathrm{~kg}$. Peak $\mathrm{Fz}$ was significantly $(p<.001)$ higher during paced (1659 $N)$ than unpaced $(1507 \mathrm{~N})$ trials. The Speed $\times$ Method interaction was also significant, with larger increases in $F z$ occurring for arm $(215 \mathrm{~N})$ and manual $(211 \mathrm{~N})$ than hoist $(106 \mathrm{~N})$ transfers of comparable mass (Figure 3). Although not significant, the influence of mass appears to be less consistent for hoist-assisted transfers (Figure 3). Muscular antagonism was significantly $(p<.05)$ higher during paced $(809 \mathrm{~N})$ than unpaced $(705 \mathrm{~N})$ trials.

\section{DISCUSSION}

Material handling systems are attractive as an intervention strategy to alleviate musculoskeletal stresses otherwise present during manual operations. Intuitive benefits of these devices arise from the reduction in static (gravitational) loads that must be balanced by the operator. However, given anecdotal reports that material handling systems (of the manipulator type) have not been widely accepted, as well as the potentially adverse productivity and process decrements resulting from manipulator-assisted slowdown, a basic experimental study was undertaken to ascertain the effects of pacing during object transfers.

Results demonstrated that increased task execution speed $(20 \%$ average decrease in transfer time) caused increases in peak hand forces, trunk kinematics, lumbar moments, lumbar spine forces, and muscular antagonism. The paced transfers were achieved using peak hand forces that were $\sim 10 \%$ higher than baseline values, smaller than the effects observed by Danz and Ayoub (1992) for manual lifting at natural and "fast as possible" speeds. The most substantial effects of pacing in torso kinematics were seen in the frontal and horizontal plane angular velocities, each of which increased $\sim 10 \%-15 \%$ during the paced trials. Larger peak lumbar moments were generated during the paced trials, which is consistent with other work (e.g., Bush-Joseph, Schipplein, Andersson, \& Andriacchi, 1988) showing that peak moments are proportional to lifting speed. The $20 \%$ increase in pace was associated with increased peak spine forces of $\sim 10 \%$ and increased muscular antagonism of $\sim 17 \%$.
If it is assumed that the risk of musculoskeletal injury is at least monotonically related to the magnitudes of either external (hand forces and lumbar moments) or reactive (spine compression and shear) loads, then these results suggest a direct relationship between pacing and injury potential. Some caution is warranted, however, because research has yet to demonstrate a quantitative relationship between specific musculoskeletal (e.g., spine) forces and injury. At this point, results at least imply that use of a material handling manipulator, under conditions that require work to be performed at a faster than "preferred" pace, will cause increased musculoskeletal stresses and probably increases in injury risk.

Justification for this conclusion takes several forms. First, earlier results (Nussbaum, Chaffin, Stump, et al., 1998) demonstrated that the use of a manipulator in self-paced tasks required hand forces that, on the basis of psychophysically derived limits, would be acceptable to most of the general population. The increase in peak hand forces that were found during paced trials may be sufficient to cause some tasks to be unacceptable and, given the traditional implicit interpretation of psychophysical limits, increase the likelihood of injury. Second, torso kinematics (postures and motion characteristics) have been implicated as injury risk factors (e.g., Marras et al., 1993). Although the increases in torso angular deviations and angular velocities observed in this study were relatively small, it is likely nonetheless that additional injury risk is imposed. Third, the paced tasks required higher levels of lumbar moments. Although again these increases were somewhat small overall, it does suggest that a larger fraction of the population would not have sufficient strength to perform the tasks, thus decreasing job accessibility and increasing musculoskeletal risk (Battié et al., 1989; Chaffin, Herrin, \& Keyserling, 1978; Snook, 1987). Fourth, the paced trials imposed higher levels of both compression and shear force on the lumbar spine. Given the existing evidence relating spine forces to failure of vertebrae and discs (e.g., Adams \& Dolan, 1995; McGill, 1997), further evidence of increased injury risk is indicated.

It must be cautioned, despite these arguments, that the results may not extrapolate 
beyond the level of forced pacing achieved in the study $($ mean $=20.2 \%$, range $=5 \%-50 \%$ ). It is expected, given the large inertia of many commercially available material handling manipulators, that larger biomechanical effects of pacing would result than those documented here, especially on spinal loads, when tasks are performed more rapidly than required in this study.

When using the manipulators, even at the faster speed, most biomechanical measures remained well below those obtained from manual operations at slower, preferred speeds (Figure 2). However, during the asymmetric (lateral) transfers, using the manipulator at a faster speed resulted in spine compression forces that approached and lateral shear forces that exceeded those seen in manual transfers (Figure 3). Thus although it can generally be concluded that faster-than-normal operation of a manipulator is less physically stressful than self-paced manual work, there may be conditions for which the use of a manipulator is actually more stressful and potentially injurious.

Previous studies have shown that torso muscle antagonism is present during trunk motions and lifting activities (e.g., Thelen, Schultz, \& Ashton-Miller, 1995), with suggestions that antagonism substantially increases spine forces (Cholewicki, McGill, \& Norman, 1995; Granata \& Marras, 1995b; Hughes, 1991). In the present work, a metric was employed to quantify the extent of muscular antagonism by determining the difference between lumbar compression forces predicted by an EMG-based model and an optimizationbased approach, and peak values increased approximately $17 \%$ overall during paced trials. It appears that antagonism is increasingly incorporated as a motor strategy with more rapid transfer speed. As antagonism is associated with increased metabolic demands, it can be expected that the relation between pacing and energy expenditure will be nonlinear.

When material handling manipulators were used at faster-than-preferred speeds, there were both behavioral (hand forces, trunk kinematics) and biomechanical (muscle and spine forces) changes. Participants appeared to select motor strategies to achieve more rapid task execution that involved primarily the use of higher hand forces while maintaining a relatively consistent postural sequence. Average values of hand forces were also seen to consistently increase (not reported here), which is expected because the various masses (objects and manipulators) required larger or more prolonged accelerations during the paced trials. The use of a strategy that involves a higher degree of upper extremity effort can explain several of the observed results. The arms are used to accelerate, propel, and decelerate the objects, while at the same time the trunk is maintained relatively rigid with respect to the pelvis and lower extremities. In the paced trials, larger hand forces are achieved through increased moments generated at the elbow and shoulder, leading in turn to elevated lumbar moments. These efforts require a base of support that is relatively more rigid, a need that is consistent with the observed increases in torso muscle antagonism and with observations that participants performed the paced trials with what appeared to be a more rigid stance and greater arm effort.

The effects of pacing on hand forces and lumbar muscle antagonism were generally larger when using the hoist and arm than when using manual transfers. These increases are easily explained in the case of arm transfers, because this device has a large mass and thus requires larger forces to achieve acceleration. The articulated arm, essentially a two-segment linkage, also has a complex inertia wherein the forces required for acceleration can change substantially with the configuration of the linkage. It is likely that a learning process is involved in adapting to this device and using it efficiently. There is evidence that such learning occurs when using manipulators (Chaffin, Stump, Nussbaum, \& Baker, 1999), though it probably occurs over many repetitions.

The hoist also requires a level of coordination, as evidenced by the larger hand forces and lumbar muscle antagonism seen, despite the relatively low intrinsic mass of the device. In part, elevated hand forces are needed to slide the hoist on its overhead bridge rail system. The hoist, if viewed as a complex pendulum, should have natural frequency and should require more effort to use at a rate higher than this frequency. Assuming that par- 
ticipants determined this natural pace during practice trials, then the relatively larger effort increment required during paced trials would be explained by exceeding the natural frequency. Further evidence is seen in the differential effect of mass when using the hoist (see Figure 3).

Both the arm and hoist also have comparable natural speeds in the vertical direction, resulting from the intrinsic speed of the pneumatic lift mechanisms. During the paced experimental trials, participants were often observed attempting to "force" the devices on and off the platforms in order to keep up with pacing tones. These indications of relatively higher coordinative demands imposed by the use of manipulators suggest that their implementation will be associated with higher levels of musculoskeletal stress and that time should be allowed for skill acquisition or that training and practice should be provided.

This study indicates that load manipulators exert a complex dynamic effect on musculoskeletal functions, especially when a worker must move faster than his or her chosen or preferred pace. Though the results are limited to a laboratory study with relatively moderate loads, they substantiate the need for great care in designing and operating these systems, especially if a faster-than-preferred pace is required.

\section{ACKNOWLEDGMENTS}

All experiments were performed in the Center for Ergonomics at the University of Michigan. The authors wish to thank Gerri Baker and Ben Stump for their assistance in data collection and analysis. This work was partially supported by Ford Motor Company, in cooperation with Catherine Sowden, Brad Joseph, and Glenn Jimmerson.

\section{REFERENCES}

Adams, M. A., \& Dolan, P. (1995). Recent advances in lumbar spinal mechanics and their clinical significance. Clinical Biomechanics, 10, 3-19.

Ayoub, M. A. (1982). Control of manual lifting hazards: Training in safe handling. Journal of Occupational Medicine, 24, 573-577.

Battié, M. C., Bigos, S. J., Fisher, L. D., Hansson, T. H., Jones, M. E., \& Wortley, M. D. (1989). Isometric lifting strength as a predictor of industrial back pain reports. Spine, 14, 851-856.
Bean, J. C., Chaffin, D. B., \& Schultz, A. B. (1988). Biomechanical model calculations of muscle contraction forces: A double linear programming method. Journal of Biomechanics, 21, 59-66.

Bush-Joseph, C., Schipplein, O., Andersson, G. B. J., \& Andriacchi, T. P. (1988). Influence of dynamic factors on the lumbar spine moment in lifting. Ergonomics, 31, 211-216.

Chaffin, D. B., Herrin, G. D., \& Keyserling, W. M. (1978). Preemployment strength testing. Journal of Occupational Medicine, 20, 403-408.

Chaffin, D. B., \& Park, K. S. (1973). A longitudinal study of lowback pain as associated with occupational lifting factors. American Industrial Hygiene Association Journal, 34, 513-525.

Chaffin, D. B., Stump, B. S., Nussbaum, M. A., \& Baker, G. (1999). Low back stresses when learning to use a material handling device. Ergonomics, 42, 94-110.

Cholewicki, J., McGill, S. M., \& Norman, R. W. (1995). Comparison of muscle forces and joint load from an optimization and EMG assisted lumbar spine model: Towards development of a hybrid approach. Journal of Biomechanics, 28, $321-331$.

Danz, M. E., \& Ayoub, M. M. (1992). The effects of speed, frequency, and load on measured hand forces for a floor to knuckle lifting task. Ergonomics, 35, 833-843.

Granata, K. P., \& Marras, W. S. (1993). An EMG-assisted model of loads on the lumbar spine during asymmetric trunk extensions. Journal of Biomechanics, 26, 1429-1438.

Granata, K. P., \& Marras, W. S. (1995a). An EMG-assisted model of trunk loading during free-dynamic lifting. Journal of Biomechanics, 28, 1309-1317.

Granata, K. P., \& Marras, W. S. (1995b). The influence of trunk muscle coactivity on dynamic spinal loads. Spine, 20, 913-919.

Hughes, R. E. (1991). Empirical evaluation of optimization-based lumbar muscle force predictions models. Unpublished doctoral dissertation, University of Michigan, Ann Arbor.

Lavender, S. A., Tsuang, Y. H., Andersson, G. B. J., Hafezi, A., \& Shin, C. C. (1992). Trunk muscle cocontraction: The effects of moment direction and moment magnitude. Journal of Orthopaedic Research, 10, 691-700.

Marras, W. S., Lavender, S. A., Leurgans, S. E., Rajulu, S. L., Alread, W. G., Fathallah, F. A., \& Furguson, S. A. (1993). The role of dynamic three-dimensional trunk motion in occupationally related low back disorders: The effects of workplace factors, trunk position and trunk motion characteristics in the risk of injury. Spine, 18, 617-628.

McGill, S. M. (1992). A myoelectrically based dynamic threedimensional model to predict loads on the lumbar spine tissues during lateral bending. Journal of Biomechanics, 25, $395-414$.

McGill, S. M. (1997). The biomechanics of low back injury: Implications on current practice in industry and the clinic. Journal of Biomechanics, 30, 465-475.

Nussbaum, M. A., \& Chaffin, D. B. (1996). Development and evaluation of a geometric model of the human torso. Clinical Biomechanics, 11, 25-34.

Nussbaum, M. A., \& Chaffin, D. B. (1998). Lumbar muscle force estimation using a subject-invariant 5-parameter EMG-based model. Journal of Biomechanics, 31, 667-672.

Nussbaum, M. A., Chaffin, D. B., \& Baker, G. (in press). Biomechanical analysis of material handling manipulators in short distance transfers of moderate mass objects: Joint strength, spine forces, and muscular antagonism. Ergonomics.

Nussbaum, M. A., Chaffin, D. B., Stump, B. S., Baker, G., \& Foulke, J. (1998). Motion times, hand forces, and trunk kinematics when using material handling manipulators in short distance transfers of moderate mass objects. Manuscript submitted for publication.

Nussbaum, M. A., Zhang, X., \& Chaffin, D. B. (1999). Heuristics for locating upper extremity joint centers from surface markers: Empirical derivation, evaluation, and optimization-based enhancement. Manuscript submitted for publication.

Resnick, M. L., \& Chaffin, D. B. (1996). Kinematics, kinetics, and psychophysical perceptions in symmetric and twisting pushing and pulling tasks. Human Factors, 38, 114-129. 
Snook, S. H. (1987). Approaches to preplacement testing and selection of workers. Ergonomics, 30, 241-247.

Sowden, C., Jimmerson, G., Joseph, B., \& Chaffin, D. B. (1998). Material and part handling in manufacturing. In P. Mitchell (Ed.), Tool and manufacturing engineers handbook (4th ed., pp. 7-1-7-7). Dearborn, MI: Society of Manufacturing Engineers.

Thelen, D. G., Schultz, A. B., \& Ashton-Miller, J. A. (1995). Cocontraction of lumbar muscles during the development of time-varying triaxial moments. Journal of Orthopaedic Research, 13, 390-398.

Winter, D. A. (1990). Biomechanics and motor control of human movement (2nd ed.). New York: Wiley.

Woldstad, J. C., \& Chaffin, D. B. (1994). Dynamic push and pull forces while using a manual material handling assist device. IIE Transactions, 26, 77-88.

Woldstad, J. C., \& Reasor, R. J. (1996). Manual materials assist devices. In A. Bhattacharya \& J. D. McGlothlin (Eds.), Occupational ergonomics: Theory and applications (pp. 351-365). New York: Dekker.
Maury A. Nussbaum is an assistant professor in the Industrial and Systems Engineering Department at Virginia Polytechnic Institute and State University and Director of the Industrial Ergonomics Laboratory. He received his Ph.D. in industrial and operations engineering from the University of Michigan in 1994.

Don B. Chaffin is the G. Lawton and Louise G. Johnson Professor of Industrial and Operations Engineering and Occupational Health and Director of the Center for Ergonomics at the University of Michigan. He received his Ph.D. in industrial engineering from the University of Michigan in 1967.

Date received: May 8, 1998

Date accepted: October 23, 1998 